Supporting information

\title{
Determination of Nonlinear Optical Efficiencies of Ultrahigh- $Q$ Photonic Crystal Nanocavities with Structural Imperfections
}

\author{
Heungjoon Kim, ${ }^{\dagger, *}$ Susumu Noda,${ }^{*}$ Bong-Shik Song, ${ }^{\dagger, *}, *$ and Takashi Asano ${ }^{\ddagger}, *$ \\ †Department of Electrical and Computer Engineering, Sungkyunkwan University, Suwon \\ 16419, Republic of Korea \\ * Department of Electronic Science and Engineering, Kyoto University, Kyoto 615-8510, \\ Japan \\ *Corresponding authors: Bong-Shik Song (songwiz@skku.edu) and Takashi Asano \\ (tasano@qoe.kuee.kyoto-u.ac.jp)
}

This PDF file includes (total number of pages 5):

- $\quad$ Supplements S1 to S3

- $\quad$ Figures S1 to S3

- References S1 and S2 


\section{S1. Additional real space images of radiation components}

(a)

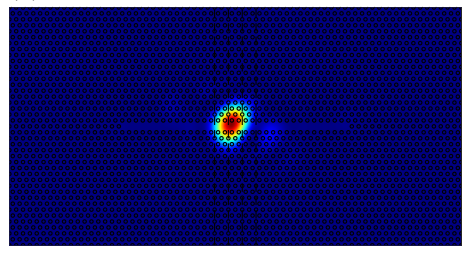

(b)

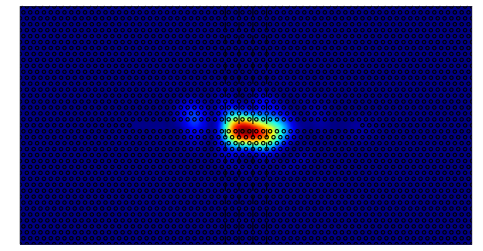

(c)

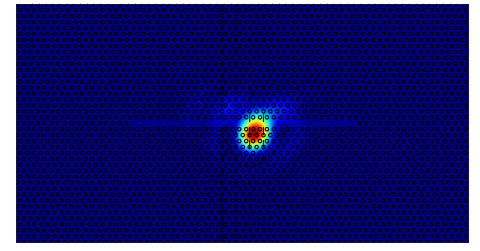

Figure S1. The calculated real space images of radiation components of the imperfect (a) $5^{\text {th }}$, (b) $15^{\text {th }}$, and (c) $30^{\text {th }}$ cavities for fundamental light in Figure $2 \mathrm{~b}$.

Figure S1 shows the images calculated for cavities with other random imperfection patterns, where an intense single spot is always observed at around the center of each cavity. These images are largely different from that of the cavity without imperfection shown in Figure $2 b$, which consists of two intense radiation spots at both edges of the cavity and four weak symmetric spots at around the center of the cavity. It is because the light leakages from imperfect cavities with an ultrahigh- $Q$ design are almost determined by the light scattering due to the structural imperfections. In this case, the intensity of such scattering is highest at around the center of the cavity where the cavity mode field intensity is the highest, resulting in an intense single spot at around the center of the cavity. In contrast, the light leakages from perfect cavities with an ultrahigh- $Q$ design are suppressed by the design itself, and residual components which could not be cancelled out by the design determine the shape of the spots. Therefore, there is a large difference between the imperfect and perfect cavity in the case of fundamental mode, and imperfect cavities almost show single spot at the center. 


\section{S2. Collection efficiencies ( $c_{\mathrm{w}}$ and $c_{\mathrm{v}}$ ) for various lens' $\mathbf{N A}$}

(a)

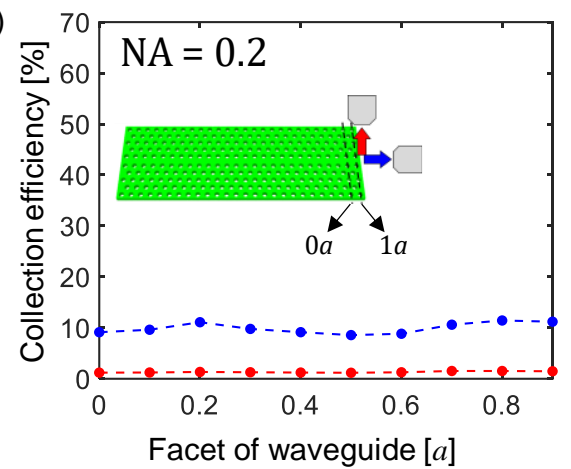

(c)

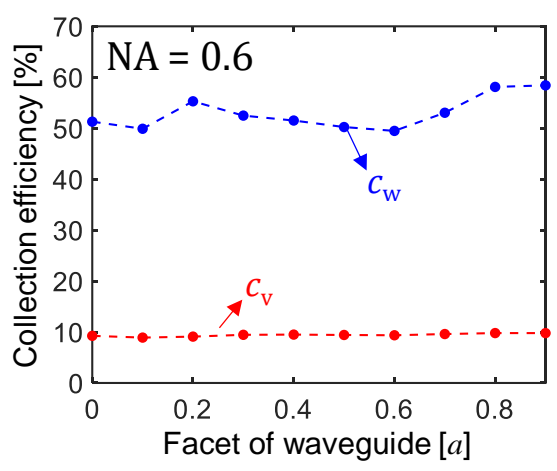

(b)

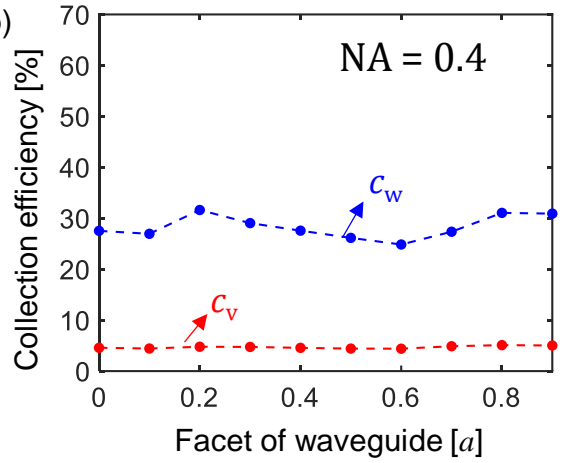

(d)

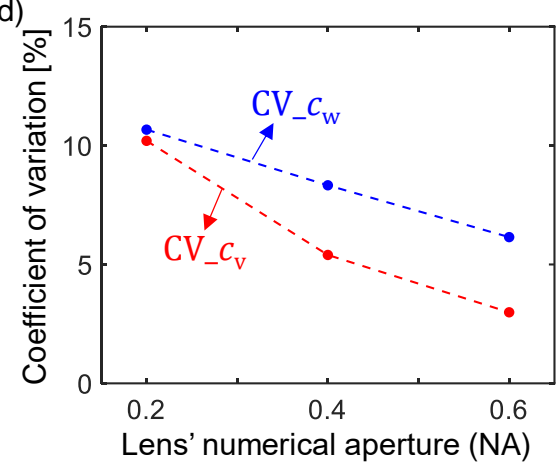

Figure S2. (a,b,c) Calculated lens collection efficiencies $\left(c_{\mathrm{w}}\right.$ and $\left.c_{\mathrm{v}}\right)$ of the waveguide-directional transmitted light and transmitted-vertical-emitted light for various facets of the photonic crystal waveguide and NA. (d) Summarized coefficients of variation of $c_{\mathrm{w}}$ and $c_{\mathrm{v}}$ for various lens' NA.

We calculated the collection efficiencies $\left(c_{\mathrm{w}}\right.$ and $\left.c_{\mathrm{v}}\right)$ of the waveguide-directional transmitted light and transmitted-vertical-emitted light for various lens NA $(0.2,0.4$, and 0.6). As seen in Figure $\mathrm{S} 2 \mathrm{a}, \mathrm{b}$, and $\mathrm{c}$ both collection efficiencies of $c_{\mathrm{w}}$ and $c_{\mathrm{v}}$ increase with NA, and it was found that $c_{\mathrm{W}}$ has a larger dependence on NA. To analyze statistically the influence of NA on the variation, we calculated the dependence of the coefficients of variation $\left(\mathrm{CV} c_{\mathrm{W}}\right.$ and $\left.\mathrm{CV} \_c_{\mathrm{V}}\right)$ on NA, as shown in Figure S2d. It is seen that variations of both $c_{\mathrm{w}}$ and $c_{\mathrm{V}}$ decrease with NA, and the variation of $c_{\mathrm{v}}$ is smaller than that of $c_{\mathrm{w}}$. Therefore, it is concluded that the observation of the transmitted-vertical-emitted light provides more reliable estimation even if we changed the NA of the lens. 


\section{S3. Transmittance and power in the cavity}

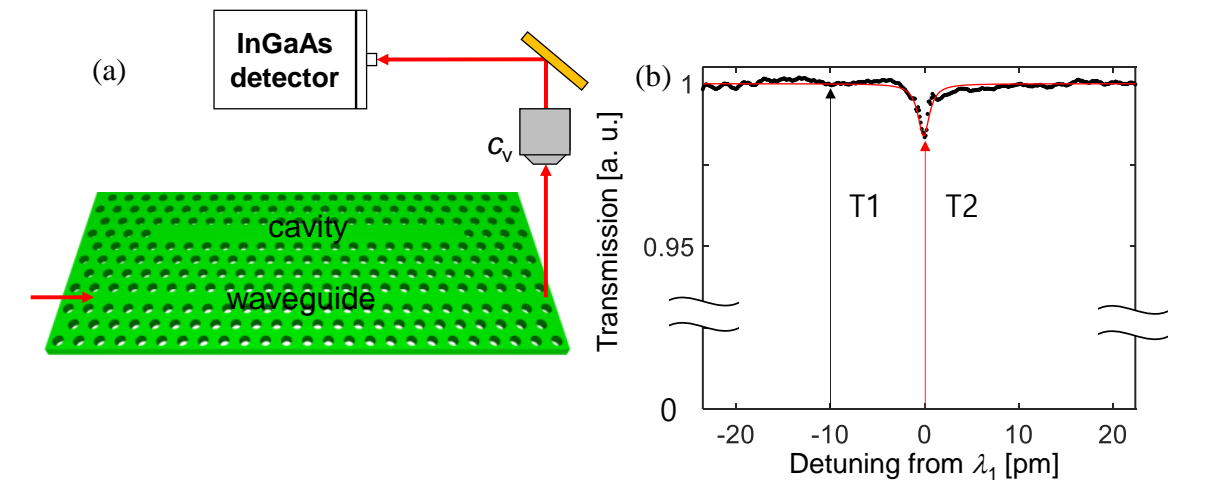

Figure S3. (a) Schematic of optical setup for measurement of transmitted-vertically-emitted light. (b) Detuning spectrum of the measurement from the resonant wavelength $\lambda_{1}=1503.5 \mathrm{~nm}$ of Figure $1 \mathrm{c}$.

As seen in the spectrum, there is a dip near the detuned wavelength of zero $\left(=\lambda_{1}\right)$ (here, it is noteworthy that the linewidth of the dip spectrum is exactly equal to that of the resonant spectrum of Figure 1c). Furthermore, the transmittance $\left(T_{\text {dip }}\right)$ at the resonant wavelength is calculated and the (1- $\left.T_{\text {dip }}\right)$ implies the amount of light coupled to the cavity. Then power $\left(P_{\text {in }}\right)$ coupled to (inside) the cavity the cavity is estimated as ( $c_{\mathrm{v}}$ and $P_{\text {detector }}$ are the vertical coupling efficiency and power measured by detector, respectively). Similar technique of employing the dip spectrum is used in the estimation of add/drop filter efficiency ${ }^{\mathrm{S} 1}$ and intrinsic $Q$ factors of photonic crystal cavities ${ }^{\mathrm{S} 2}$. 


\section{References}

(S1) Asano, T.; Song, B. S.; Tanaka, Y.; Noda, S.; Investigation of a channel-add/drop-filtering device using acceptor-type point defects in a two-dimensional photonic-crystal slab. Appl. Phys. Lett. 2003, 83, 407.

(S2) Akahane, Y.; Asano, T.; Song, B. S.; Noda, S.; Fine-tuned high- $Q$ photonic-crystal nanocavity. Opt. Express 2005, 13, 1202-1214. 